\title{
DATA ASSOCIATION TECHNIQUES FOR NEAR-CONTEMPORANEOUS SAR AND AIS DATASETS FROM NOVASAR-1
}

\author{
Maximilian Rodger and Raffaella Guida \\ Surrey Space Centre, University of Surrey, Guildford, UK \\ m.rodger@surrey.ac.uk
}

\begin{abstract}
In this research the best techniques of fusion for nearcontemporaneous Synthetic Aperture Radar (SAR) and Automatic Identification System (AIS) datasets are studied to simulate the expected performance from NovaSAR-1. Specifically, data association techniques are quantitatively compared by performing a series of Monte Carlo tests. The evaluation has been carried out using a satellite-based AIS dataset acquired from the English Channel on 07 June 2016, and SAR ship detections are simulated by reckoning the AIS dataset forward in time along a geodesic on a WGS84 reference ellipsoid. Accurate data association is achieved using an $m$-best multidimensional assignment technique, which is consistent with being used in an operational environment, especially in high-density shipping areas.
\end{abstract}

Index Terms - synthetic aperture radar, SAR, automatic identification system, AIS, data fusion, data association, space-based maritime surveillance, NovaSAR-1

\section{INTRODUCTION}

Space-based maritime surveillance has recently seen growing interest on an international scale, partly due to its global coverage capability and its independence of most meteorological conditions provided by Synthetic Aperture Radar (SAR). With the aid of the Automatic Identification System (AIS), the combination of information from these two systems provides the possibility of a global maritime surveillance system. The AIS is a line-of-sight method of communication which provides and exchanges ship information (e.g. identity, position, speed, dimensions, etc.) with other ships, shore-based stations and recently satellites in low-Earth orbit (LEO) [1]. The advantage of fusing data from SAR and AIS sensors is the increased ship detection probability. For example, cooperative ships can be detected thanks to the self-reporting data of the AIS, and non-cooperative ships, that have either lost their AIS signal or are intentionally avoiding detection to perform illicit activities, can be detected thanks to the independent, observation-based data of the SAR sensor (see Fig. 1). This is of great significance to the maritime community, especially

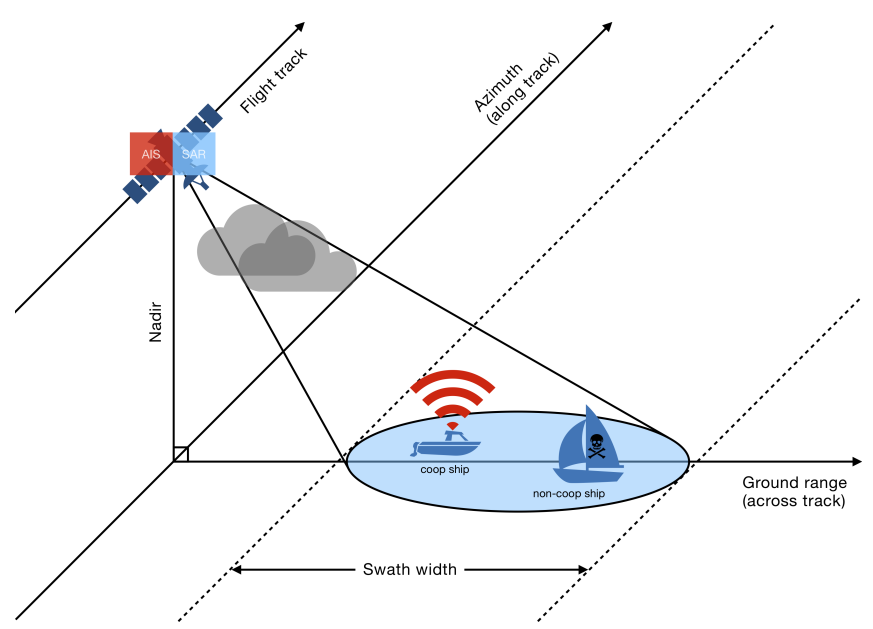

Fig. 1: Conceptual overview of a space-based maritime surveillance system with co-located SAR and AIS sensors.

those involved in maritime safety and security, providing a comprehensive picture of human activities at sea.

In areas of research which utilise data fusion techniques, the core challenge is the spatial and temporal matching of datasets, especially if their data types are heterogeneous. The ability to reliably associate SAR and AIS datasets largely comes down to the temporal difference or "temporal gap" between corresponding sensor acquisitions. With the UK's first spaceborne SAR mission, NovaSAR-1 [2], the challenge of fusion with temporally diverse data is thought to be eliminated due to the SAR and AIS sensors being co-located on the same platform. However, a further challenge is the data association of SAR and AIS datasets in high-density shipping areas. The data association step is the cornerstone of the surveillance system and is the process of linking measurements, such as SAR ship detections and AIS observations, deemed to be of a common origin. Ships detected in SAR imagery can be wrongly associated with AIS observations in congested areas (e.g. near ports and shipping lanes) and result in an erroneous and/or inaccurate maritime picture. The data association of SAR and AIS datasets has been predominately carried out using the well-known nearest neighbour (NN) technique (e.g. [3-5]). The attraction of the NN method is its 
simplicity, both conceptually and computationally, where associations are made based on the minimum distance between SAR ship detections and AIS observations. While appropriate for a single target or widely separated targets (i.e. open ocean), its performance degrades rapidly in closely-spaced or dense situations in terms of the level of correspondence of the data. As such, the NN technique and its variants are suboptimal in addressing the problem of SAR and AIS data association in high-density shipping areas. Unconventional techniques which aim to solve the above problem include the global nearest neighbour (GNN) [6] and point set registration [7] techniques, which are the motivation for the next section.

\section{METHODOLOGY}

In this section, solutions to the data association problem are discussed. The problem of SAR and AIS data association is first described in terms of the optimal assignment problem, where the distance for each SAR-AIS pair is computed and the associations (or "assignments") are made by minimising the sum of the total distances for all possible pairings. This technique is used extensively in multitarget tracking (MTT) and is often referred to as the global nearest neighbour (GNN) approach to data association [8]. This technique is then extended to establish an $m$-best multidimensional assignment technique. Next, the association of SAR and AIS point targets are treated similar to the registration of point sets, which is a common problem found in computer vision where the correspondences between the point sets are unknown a priori.

\subsection{The 2-D assignment problem}

The 2-D assignment problem is a special type of optimisation problem which was first considered in economics, where common problems included assigning personnel to jobs and delivery vehicles to locations. The objective in these problems is to minimise the cost (or maximise the profit) using the available resources. Initially, linear programming techniques $[9,10]$ were used to solve the assignment problem, but as the assignment problem became relevant to other fields such as tracking, improved techniques evolved [11]. In the example of assigning personnel to jobs, assuming the total number of people and jobs are equal, the objective function for the assignment problem can be represented as

$$
\min \sum_{i=1}^{n} \sum_{j=1}^{n} C_{i j} x_{i j}
$$

subject to

$$
\sum_{j=1}^{n} x_{i j}=1, \quad i=1, \ldots, n
$$

$$
\sum_{i=1}^{n} x_{i j}=1, \quad j=1, \ldots, n
$$

where $C_{i j}$ is a matrix whose elements give the cost of assigning the $i$ th person to the $j$ th job, $x_{i j}=1$ if job $j$ is assigned to person $i$ and $x_{i j}=0$ otherwise. The constraints shown in Eq. 2 and Eq. 3 indicate that for each job only one person can be assigned (and vice versa), i.e., a one-to-one constraint. In the context of SAR and AIS data association, the $x_{i j}$ elements are the SAR-AIS pairs to be found and the cost (total distance) is that which is to be minimised. The cost function can include not only positional information but kinematic and static information derived from SAR and reported by AIS (e.g. the ship's speed, heading, width and length) to enhance the results. Similar to the first example, the assignment is subject to the same one-to-one constraint, i.e., only one SAR ship detection can be assigned to one AIS data point or track and vice versa. In this paper, the Jonker and Volgenant (JV) algorithm [11] is selected to provide an optimal solution to the assignment problem.

\subsection{The $m$-best 2 -D assignment problem}

In the $m$-best 2-D assignment problem, not only is the single best assignment found but also the top $m$-best assignments (rank-ordered) [8]. The advantage of using an $m$-best technique over a single-best technique is that an additional (or auxiliary) assignment can occur for the $m$-best assignments based on kinematic and static information derived from SAR and reported by AIS. This multidimensional approach to data association allows for an improved confidence level in the SAR-to-AIS assignments.

\subsection{Geometric alignment}

Here, the data association is formulated as a point set registration problem, which is based on the RANdom SAmple Consensus (RANSAC) algorithm [12]. The RANSAC algorithm is a robust method, able to deal with a large proportion of outliers, which returns a 2-D geometric or spatial transformation that optimally aligns SAR ship detections to AIS-reported positions. Specifically, the algorithm can determine a similarity, affine or projective transformation of the two-dimensional AIS positions (i.e. latitude and longitude coordinates) to best conform them to the SAR positions.

\section{RESULTS}

The performance and feasibility of the data association techniques discussed above are now quantitatively compared by performing a series of Monte Carlo tests. 


\subsection{Data description}

\subsubsection{AIS dataset}

The AIS dataset is extracted from data provided by exactEarth Ltd, which was acquired from the English Channel on 07 June 2016. The data is first filtered to obtain 107 unique ships (point targets) with non-zero dimensions and speed, where tracks and ships anchored in port are not considered. The mean, $\mu$, and standard deviation, $\sigma$, are calculated for the speed over ground (SOG), length and width data fields. For the strongly non-uniform heading data, the median and interquartile range (IQR) are given. Histograms are shown in Fig. 2 below which can be used to simulate the SAR ship detections (described next).

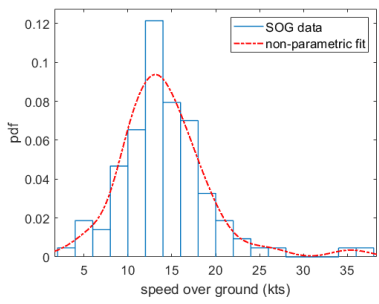

(a) $\mu=14.2 \mathrm{kts} ; \sigma=5.2 \mathrm{kts}$

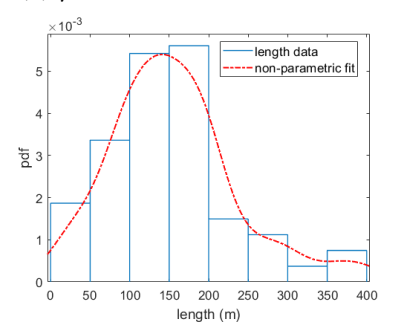

(c) $\mu=155 \mathrm{~m} ; \sigma=80 \mathrm{~m}$

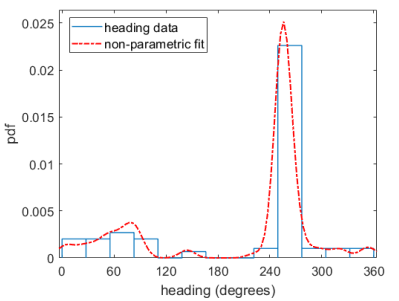

(b) median $=252^{\circ} ; \mathrm{IQR}=174^{\circ}$

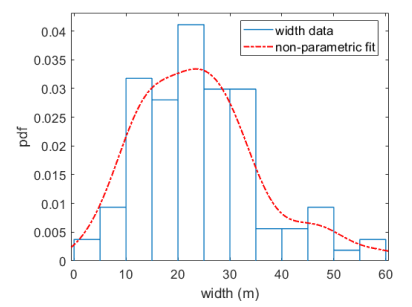

(d) $\mu=24 \mathrm{~m} ; \sigma=12 \mathrm{~m}$
Fig. 2: Histograms for the (a) SOG (b) heading (c) length and (d) width data derived from the AIS dataset, with respective statistical values. For each histogram, the probability density function (pdf) of the kernel distribution is fitted to the data.

\subsubsection{SAR dataset}

To simulate SAR ship detections, the AIS dataset is reckoned forward in time. The reckoning process calculates new positions along a geodesic on a WGS84 reference ellipsoid assuming constant speed and heading. It is assumed that the speed and heading of a ship remains relatively constant when the reckoning time is on the order of a few minutes. By reckoning the AIS dataset forward in time, the performance of the algorithms can be measured as a function of the temporal difference (or gap) between the sensors' acquisition time.

\subsection{Performance of algorithms}

A series of Monte Carlo tests are performed to measure the performance and robustness of the algorithms. The perfor- mance of the algorithms are compared by defining the assignment accuracy, which is the percentage of correct correspondences between the two datasets. Because the data are simulated, the correct correspondences are known a priori. To produce the random element in the Monte Carlo tests, the speed and heading for each ship are changed every iteration according to the statistical distributions derived from the AIS dataset (see Fig. 2). Fig. 3 and Fig. 4 show the assignment accuracy as a function of the temporal gap, $\Delta T$, and number of ships, $n$, respectively, for the NN, JV and RANSAC algorithms ${ }^{1}$. The measured assignment accuracy of the respective algorithms is based first on position, where the distance metric used to populate the cost matrix (Eq. 1) is the geodesic distance between the SAR and AIS positions (assuming a WGS84 reference ellipsoid). Additionally, for all algorithms an equal amount of SAR and AIS data points are considered.

The JV algorithm is then extended to an $m$-best technique where the 3-best assignments (i.e. $m=3$ ) are found for a given ship. For each misassignment in the top-ranked results, its reported length and width are compared to the next two best assignments' length and width; if there is a correct match to either the second or third best assignment, the misassignment is updated to the correct result. The $m$-best technique is also evaluated for unequal SAR and AIS data points (dashed line in Fig. 3), where a third of the AIS data points are removed. The algorithm maintains a high assignment accuracy in the presence of missing data and SAR ship detections with no correspondence are flagged as 'unassigned'.

The assignment-based techniques (GNN) such as the JV algorithm offer the best performance, both in terms of association accuracy and computational performance. In particular, the $m$-best multidimensional assignment technique outperforms conventional techniques found in the literature on average by $11 \%$ in terms of association accuracy and baseline GNN algorithms are over 40 times faster in execution time ${ }^{2}$. In contrast, the RANSAC algorithm performs poorly in terms of average assignment accuracy. It is conjectured that common transformations such as similarity, affine and projective transformations are ill-suited in describing the trajectory of a fleet of ships for a temporal gap greater than a few minutes. Moreover, by using a multidimensional approach, i.e., using auxiliary information such as ships' heading, dimensions, etc. a greater level of correspondence of the data is achieved and non-cooperative or "rogue" shipping can be better identified. The $m$-best multidimensional assignment technique is consistent with being used in an operational environment, especially in high-density shipping areas, where the execution time is independent of $\Delta T$ and follows a second order polynomial fit with $n$. Specifically, the execution time is consistently less than $0.5 \mathrm{~s}$ for a $\Delta T$ of $0-10$ mins and ranges from approximately $0.01-8 \mathrm{~s}$ for $4-107$ ships in the scene.

\footnotetext{
${ }^{1}$ All algorithms have been implemented in the MATLAB ${ }^{\circledR}$ environment

${ }^{2} \mathrm{CPU}$ : Intel ${ }^{\circledR} \mathrm{Core}^{\mathrm{TM}}$ i5-6500@ $3.20 \mathrm{GHz}$ (quad core).
} 


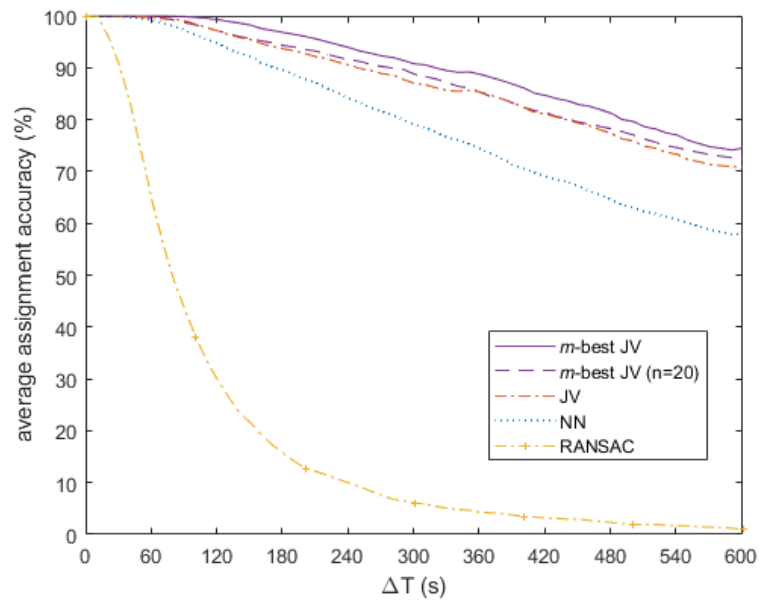

Fig. 3: Average assignment accuracy of the respective algorithms as a function of the temporal gap, $\Delta T$, for $n=30$. For each increment (i.e. for every $10 \mathrm{~s}$ ), the assignment accuracy results comprise an average over $10^{2}$ iterations.

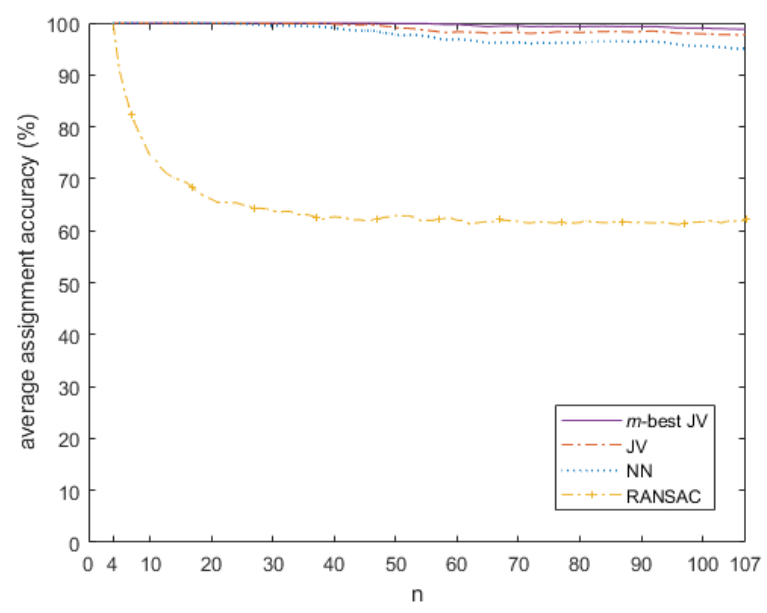

Fig. 4: Average assignment accuracy of the respective algorithms as a function of the number of ships, $n$, for $\Delta T=60 \mathrm{~s}$. For each increment (i.e. for every 1 ship), the assignment accuracy results comprise an average over $10^{2}$ iterations.

\section{CONCLUSIONS AND FUTURE WORK}

It is suggested that assignment-based data association algorithms should be used for the task of SAR and AIS data association for NovaSAR-1. In particular, an $m$-best technique using the JV algorithm is worth further investigation; closely spaced targets may be accurately associated using nonspatial attribute (or auxiliary) data rather than relying solely on spatial data association. In future work, the $m$-best technique will be extended to include other auxiliary information such as the kinematic information as well as improving its computational performance. Similarly, other areas worth investigating include testing different distance metrics which are used to populate the cost matrix since, as described in [8], the primary source of the computational workload depends on computing the cost coefficients of the assignment problem.

\section{REFERENCES}

[1] International Maritime Organization (IMO). (2018). International Convention for the Safety of Life at Sea (SOLAS), 1974 (Chapter V, Regulation 19, Section 2.4.5) [Online]. Available: https://tinyurl.com/qy3psge

[2] SSTL. (2018). Launched Missions: NovaSAR-1 [Online]. Available: https://tinyurl.com/y7wtmqy6

[3] R. Grasso, S. Mirra, A. Baldacci, J. Horstmann, M. Coffin and M. Jarvis, "Performance Assessment of a Mathematical Morphology Ship Detection Algorithm for SAR Images through Comparison with AIS Data," 2009 Ninth International Conference on Intelligent Systems Design and Applications, Pisa, 2009, pp. 602-607.

[4] S. K. Chaturvedi, C.-S. Yang, K. Ouchi and P. Shanmugam, "Ship Recognition by Integration of SAR and AIS," Journal of Navigation, vol. 65, no. 2, pp. 323-337, 2012.

[5] S. Voinov, E. Schwarz, D. Krause and M. Berg, "Identification of SAR Detected Targets on Sea in Near Real Time Applications for Maritime Surveillance," in Free and Open Source Software for Geospatial (FOSS4G) Conference Proceedings, vol. 16, no. 1, 2016.

[6] F. Mazzarella, M. Vespe and C. Santamaria, "SAR Ship Detection and Self-Reporting Data Fusion Based on Traffic Knowledge," IEEE Geoscience and Remote Sensing Letters, vol. 12, no. 8, pp. 1685-1689, 2015.

[7] Z. Zhao, K. F. Ji, X. W. Xing and H. X. Zou, "Effective Association of SAR and AIS Data Using Non-Rigid Point Pattern Matching," IOP Conference Series: Earth and Environmental Science, vol. 17, no. 1, pp. 012258, 2014.

[8] Y. Bar-Shalom and W. D. Blair, Multitarget-Multisensor Tracking: Applications and Advances, Volume 3. Norwood, MA: Artech House, 2000.

[9] H. W. Kuhn, "The Hungarian method for the assignment problem," Naval Research Logistics Quarterly, vol. 2, no. 1-2, pp. 83-97, 1955.

[10] J. Munkres, "Algorithms for the Assignment and Transportation Problems," Journal of the Society for Industrial and Applied Mathematics, vol. 5, no. 1, pp. 32-38, 1957.

[11] R. Jonker and A. Volgenant, "A shortest augmenting path algorithm for dense and sparse linear assignment problems," Computing, vol. 38, no. 4, pp. 325-340, 1987.

[12] M. A. Fischler and R. C. Bolles, "Random Sample Consensus: A Paradigm for Model Fitting with Applications to Image Analysis and Automated Cartography," Commun. ACM, vol. 24, no. 6, pp. 381-395, 1981. 